\title{
Análisis de la retirada del catéter peritoneal después del trasplante renal en población adulta
}

\author{
Mónica Fernández-Pérez ${ }^{1}$, Beatriz Peláez-Requejo ${ }^{1,2}$, Adela Suárez-Álvarez, Reyes Fernández-Díaz ${ }^{1,2}$, \\ Aránzazu Goncalves-Muñiz ${ }^{1}$, Miguel Núñez-Moral ${ }^{1,2,3}$ \\ ${ }^{1}$ Unidad de Gestión Clínica de Nefrología. Hospital Universitario Central de Asturias. Oviedo. España \\ ${ }^{2}$ Grupo de Investigación en Cuidados en Nefrología. Instituto de Investigación Sanitaria del Principado de Asturias \\ (ISPA). España \\ 3 Profesor asociado en Ciencias de la Salud. Universidad de Oviedo. España
}

\begin{abstract}
Como citar este artículo:
Fernández-Pérez M, Peláez-Requejo B, Suárez-Álvarez A, Fernández-Díaz R, Goncalves-Muñiz A, Núñez-Moral M. Análisis de la retirada del catéter peritoneal después del trasplante renal en población adulta.

Enferm Nefrol. 2021 0ct-Dic;24(4):417-22.
\end{abstract}

Introducción: Las guías de práctica clínica aconsejan mantener el catéter peritoneal entre 2-3 meses después del trasplante renal, cuando haya garantía de viabilidad del injerto, recomendando su retirada en el mismo acto quirúrgico en población pediátrica.

El objetivo del estudio fue comparar la necesidad de utilización del catéter peritoneal frente a las posibles complicaciones tras el trasplante renal, así como valorar la oportunidad de su retirada sistemática en el mismo acto quirúrgico.

Metodología: Se realizó un estudio descriptivo, retrospectivo entre enero de 2014 y noviembre de 2019. Incluimos a todos los pacientes mayores de 18 años en programa de diálisis peritoneal que recibieron un trasplante renal.

Resultados: Se incluyeron 124 pacientes en diálisis peritoneal con una edad media de $55,9 \pm 12,6$ años en el momento del trasplante, de los que 77 eran hombres $(62,1 \%)$. El catéter fue retirado en el momento del trasplante a 22 pacientes $(17,7 \%)$, de los cuales 2 ( $1,6 \%$ de 124 pacientes) necesitaron hemodiálisis; se retiró en un segundo tiempo a 102 pacientes (82,3\%).

\section{Correspondencia:}

Mónica Fernández Pérez

E-mail: mfp7384@hotmail.com
De ellos, 13 (10,5\% de 124 pacientes) necesitaron algún tipo de diálisis, de los que 10 (8\%) fue diálisis peritoneal. Complicaciones relacionadas con el catéter o la técnica se produjeron en 14 pacientes (13,7\%).

Conclusiones: Teniendo en cuenta que aproximadamente el $14 \%$ de los pacientes presentó complicaciones relacionadas con el catéter peritoneal y que el $92 \%$ de los pacientes trasplantados no necesitó diálisis peritoneal, valoramos como beneficioso, tanto para el paciente como para la institución, su retirada en el mismo acto quirúrgico.

PALABRAS CLAVE: trasplante renal; catéter; diálisis peritoneal; terapia de reemplazo renal; complicaciones; peritonitis.

\section{Analysis of peritoneal catheter removal after renal transplantation in adult population}

Introduction: Clinical practice guidelines advise keeping the peritoneal catheter between 2-3 months after renal transplantation, when there is a guarantee of graft viability. However, in the paediatric population, they recommend its removal during the same surgical procedure.

The aim of the study was to compare the need to use the peritoneal catheter versus possible complications 
after renal transplantation, as well as to assess the opportunity of its systematic removal in the same surgical act.

Methodology: A descriptive and retrospective study was conducted between January 2014 and November 2019. We included all patients over 18 years of age on peritoneal dialysis who received a kidney transplant.

Results: 124 patients on peritoneal dialysis with a mean age of $55.9 \pm 12.6$ years at the time of transplantation were included, of whom 77 were male $(62.1 \%)$. The catheter was removed at the time of transplantation in 22 patients $(17.7 \%)$, of whom 2 ( $1.6 \%$ of 124 patients) required haemodialysis; it was removed at a second time in 102 patients (82.3\%). Of these, 13 ( $10.5 \%$ of 124 patients) required some form of dialysis, of which $10(8 \%)$ were peritoneal dialysis. Catheter or technique-related complications occurred in 14 patients ( $13.7 \%$ ).

Conclusions: Taking into account that approximately $14 \%$ of patients had complications related to the peritoneal catheter and that $92 \%$ of transplanted patients did not need peritoneal dialysis, it was considered beneficial for both the patient and the institution to remove the catheter at the same surgical procedure.

KEYWORDS: renal transplantation; catheter; peritoneal dialysis; renal replacement therapy; complications; peritonitis.

\section{Introducción}

El momento óptimo de retirada del catéter de diálisis peritoneal en el postoperatorio del trasplante renal aún no se ha establecido. Las guías de práctica clínica aconsejan mantenerlo entre 2-3 meses, cuando haya viabilidad del injerto․ Sí parece haber más consenso en la población pediátrica, recomendando la retirada precoz tras el trasplante ${ }^{2,3}$, incluso durante el mismo ${ }^{4}$.

La persistencia del catéter una vez realizado el trasplante renal puede conllevar complicaciones, sobre todo infecciosas, como peritonitis e infección del orificio de salida ${ }^{5,6}$. En la literatura también se ha descrito el caso de perforación intestinal causada por el catéter peritoneal "durmiente ${ }^{\prime \prime 7}$, siendo sospecha de la misma la existencia de peritonitis con flora mixta. Sin embar- go, el riesgo de producirse esta complicación parece poco probable. Diversos estudios 8,9,10 intentan determinar el momento más adecuado de retirada del catéter, comparando las complicaciones y los usos posteriores del mismo; estos establecen o recomiendan periodos variables de retirada, desde 1-2 semanas hasta los 2-3 meses. Sin embargo, en lo que sí coinciden es en la necesidad de esperar a que haya garantía de buen funcionamiento del injerto. En el caso de trasplante páncreas-riñón sí que es justificada la retirada en el mismo acto ya que el páncreas es normalmente implantado en la línea media e intraperitoneal ${ }^{11}$.

En nuestro centro, el catéter peritoneal es retirado, de manera programada, una vez que se garantiza la viabilidad del injerto. Hasta que llega este momento los pacientes mantienen el autocuidado del catéter con las curas habituales del orificio de salida y los lavados periódicos del mismo, limitando al máximo las manipulaciones. En los pacientes que presentan infección del orificio de salida, el catéter es retirado en el mismo acto quirúrgico. Nos parece oportuno saber si los pacientes adultos se pueden beneficiar de la retirada durante el trasplante, evitando posibles complicaciones y la necesidad de una intervención posterior.

El objetivo del presente estudio ha sido comparar la necesidad de utilización del catéter peritoneal frente a las posibles complicaciones tras el trasplante renal, así como valorar la oportunidad de su retirada sistemática durante la cirugía de trasplante renal.

\section{Material y Método}

Se realizó un estudio descriptivo y retrospectivo entre enero de 2014 y noviembre de 2019. Incluimos a todos los pacientes mayores de 18 años en programa de diálisis peritoneal que en dicho periodo recibieron un trasplante renal.

Las variables analizadas fueron: edad en el momento del trasplante renal; sexo; tiempo en diálisis, considerado desde el inicio en domicilio hasta el trasplante renal; tiempo hasta retirada del catéter; necesidad diálisis, considerada esta como la realización de diálisis después del trasplante renal; tipos de diálisis (hemodiálisis y/o diálisis peritoneal); complicaciones relacionadas con la diálisis peritoneal.

Las variables cuantitativas fueron expresadas como medias o medianas, según siguieran una distribución normal 
Tabla 1. Total de pacientes trasplantados y agrupados según hayan realizado diálisis o no.

\begin{tabular}{|c|c|c|c|c|}
\hline & $\begin{array}{l}\text { No diálisis } \\
(\mathrm{N}=109)\end{array}$ & $\begin{array}{l}\text { Sí diálisis } \\
(\mathrm{N}=15)\end{array}$ & $\begin{array}{c}\text { Total } \\
(\mathrm{N}=124)\end{array}$ & P-value \\
\hline $\begin{array}{l}\text { Edad años } \\
\text { Media (DE) }\end{array}$ & $56,1 \quad(12,4)$ & $54,7(14,9)$ & $55,9(12,6)$ & 0,576 \\
\hline $\begin{array}{l}\text { Sexo } \\
\text { Hombre } \\
\text { Mujeres }\end{array}$ & $\begin{array}{l}64(58,7 \%) \\
45(41,3 \%)\end{array}$ & $\begin{array}{l}13(86,7 \%) \\
2(13,3 \%)\end{array}$ & $\begin{array}{l}77(62,1 \%) \\
47(37,9 \%)\end{array}$ & 0,0706 \\
\hline $\begin{array}{l}\text { T hasta Tx (sem) } \\
\text { Mediana } \\
\text { Pérdidas }\end{array}$ & $\begin{array}{c}57,3[30,8-108] \\
5(4,6 \%)\end{array}$ & $\begin{array}{c}53,1[40,4-83,7] \\
0(0 \%)\end{array}$ & $\begin{array}{c}55,9[31,7-106] \\
5(4,0 \%)\end{array}$ & \\
\hline $\begin{array}{l}\text { Tipo diálisis } \\
\text { DPA } \\
\text { DPCA }\end{array}$ & $\begin{array}{l}33(30,3 \%) \\
71(65,1 \%)\end{array}$ & $\begin{array}{c}3(20,0 \%) \\
12(80,0 \%)\end{array}$ & $\begin{array}{l}36(29,0 \%) \\
83(66,9 \%)\end{array}$ & 0,533 \\
\hline Pérdidas & $5(4,6 \%)$ & $0(0 \%)$ & $5(4,0 \%)$ & \\
\hline $\begin{array}{l}\text { Ret catéter Tx } \\
\text { NO } \\
\text { SI }\end{array}$ & $\begin{array}{l}89(81,7 \%) \\
20(18,3 \%)\end{array}$ & $\begin{array}{l}13(86,7 \%) \\
2(13,3 \%)\end{array}$ & $\begin{array}{l}102(82,3 \%) \\
22(17,7 \%)\end{array}$ & 0,907 \\
\hline $\begin{array}{l}\text { Complicaciones } \\
\text { NO } \\
\text { SI }\end{array}$ & $\begin{array}{c}100(91,7 \%) \\
9(8,3 \%)\end{array}$ & $\begin{array}{c}10(66,7 \%) \\
5(33,3 \%)\end{array}$ & $\begin{array}{c}{ }^{*} \text { Total }(\mathbf{N}=102) \\
88(86,3 \%) \\
14(13,7 \%)\end{array}$ & 0,0146 \\
\hline
\end{tabular}

DE: Desviación estándar; T hasta Tx (sem): Tiempo en semanas desde inicio en domicilio hasta el trasplante renal; DPA: Diálisis peritoneal automatizada; DPCA: Diálisis peritoneal continua ambulatoria; Pérdidas: datos no recogidos; Ret catéter Tx: Catéteres peritoneales retirados en el trasplante renal;

* Total N=102: solo se considera que pueden tener complicaciones relacionadas con la DP aquellos pacientes que conservaron el catéter después del Tx renal. po a 102 pacientes $(82,3 \%)$. El tiempo medio hasta la retirada del catéter, del grupo de retirada en segundo tiempo, fue de $147,3 \pm 69,6$ días.

En la figura 1 podemos ver el número de pacientes que necesitaron diálisis respecto al total. De los 22 pacientes a quienes habían retirado el catéter en el momento del trasplante, 2 ( $1,6 \%$ de 124 pacientes) necesitaron hemodiálisis; de los 102 pacientes a quienes no habían retirado el catéter peritoneal, 13 (10,5\% de 124 pacientes) necesitaron algún tipo de diálisis, de los que 10 ( $8 \%$ de 124 pacientes) fueron diálisis peritoneal.

Las complicaciones relacionadas con el catéter o la técnica se produjeron en 14 pacientes (ver figura 2$)$, de las que $6(5,9 \%$ de 124 pacientes) fueron peritonitis, 5 (4,9\% de 124 pacientes) infección del orificio de salida y 3 (2,9\% de 124 pacientes) otros. o no, y las cualitativas como frecuencias relativas y absolutas. Para determinar si había diferencias estadísticamente significativas se empleó el test de Mann Whitney en las variables continuas y el test de la Chi cuadrado en las categóricas. Todos los análisis se llevaron a cabo con el software $\mathrm{R}$ en su versión 4.0.2.

La investigación contó con la aprobación del Comité de Ética e Investigación del Hospital Universitario Central de Asturias.

\section{Resultados}

Se incluyeron 124 pacientes con una edad media de $55,9 \pm 12,6$ años en el momento del trasplante, de los que $77(62,1 \%)$ fueron hombres (ver tabla 1). La mediana de tiempo desde que se implantó el catéter hasta que se realizó el trasplante fue de 14 meses (Q1: 7,9-Q3: 26,5).

El catéter fue retirado en el momento del trasplante a 22 pacientes $(17,7 \%)$, retirándose en un segundo tiem-

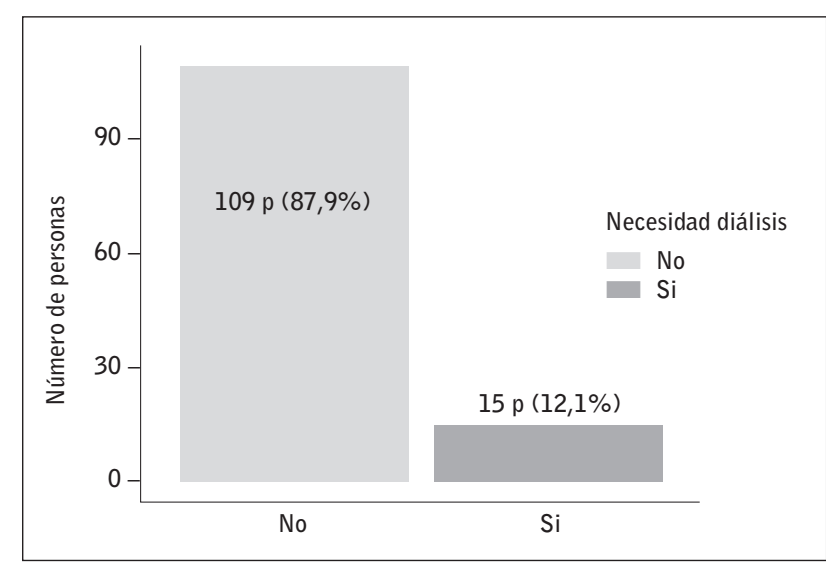

Figura 1. Distribución de los pacientes según hayan necesitado realizar diálisis o no.

\section{Discusión}

El trasplante renal es la mejor opción de terapia renal sustitutiva, esencial, viable, con buena relación coste-efectividad y que salva vidas ${ }^{12}$. Mejora la supervivencia cuando se compara con la diálisis de mantenimiento. 


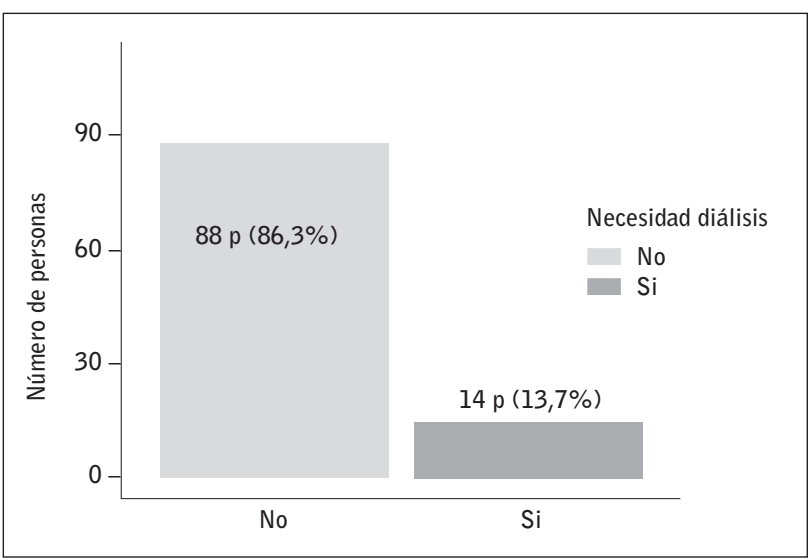

Figura 2. Distribución de los pacientes según hayan sufrido complicaciones con la diálisis peritoneal o no.

Sin embargo, hay situaciones como pueden ser complicaciones quirúrgicas, retraso en el buen funcionamiento del injerto, o un rechazo de este, hacen necesario recurrir de manera temporal o definitiva a la terapia dialítica (diálisis peritoneal y/o hemodiálisis). Es por esta razón por la cual la mayoría de los centros dejan "in situ" el catéter peritoneal hasta garantizar un buen funcionamiento del injerto $0^{8,9,10}$.

De los 124 pacientes analizados en nuestro estudio, 10 (8\%) necesitaron diálisis peritoneal después del trasplante, de los cuales 3 retornaron definitivamente a la terapia. La incidencia de complicaciones derivadas del catéter fue del $13,7 \%$. Si comparamos estos datos con los del estudio de Pérez-Fontán et $a^{\beta}{ }^{3}$, podemos observar que el $12 \%$ de sus pacientes precisaron diálisis peritoneal después del trasplante, 4 de ellos de manera indefinida. La incidencia de complicaciones fue del $15,5 \%$. Según estos resultados aconsejan esperar al $2^{\circ}$ mes postrasplante para retirada del catéter peritoneal.

Un estudio bastante parejo al nuestro en resultados es el realizado por Warren et al ${ }^{4}$, que, con 137 pacientes estudiados, un $12,7 \%$ de complicaciones postrasplante y un único paciente con necesidad de diálisis posterior, recomienda la retirada del catéter en el momento del trasplante, evitando así un posterior acto anestésico.

Mantener el buen estado del catéter peritoneal es siempre una preocupación, tanto para el paciente como para el profesional. Para mantener su permeabilidad y buen funcionamiento ante una posible necesidad de diálisis, la unidad indica al paciente la realización de lavados periódicos, administrando heparina sódica al $1 \%, 1 \mathrm{ml}$ en $2.000 \mathrm{ml}$ de solución glucosada al 1,36\% o 1,5\%, intentando reducir el número de manipulaciones para evi- tar riesgos de contaminación e infección ( 1 vez cada 10 días). Aun así, en nuestro estudio se produjeron 6 casos de peritonitis $(4,8 \%)$ en distintos pacientes. Recibieron tratamiento antibiótico y se procedió a la retirada del catéter una vez pasada la fase aguda de la infección. En el artículo de Gokal y Kost ${ }^{15}$, según su experiencia, se prescinde de los lavados del catéter y solo en el caso de dolor abdominal, fiebre 0 ante sospecha de peritonitis se hace uso del catéter. También en el estudio realizado por Bakir et al. ${ }^{5}$, se menciona la no realización de este tipo de práctica. Desconocemos si mantener estos lavados aumenta el riesgo de peritonitis, o tal vez, su no realización podría derivar en otro tipo de complicaciones como perforación intestinal. En este último estudio también se analizan posibles factores de riesgo de peritonitis tras el trasplante, factores preoperatorios como la alta incidencia de peritonitis por Staphylococcus Aureus previas al trasplante y factores postoperatorios como problemas quirúrgicos durante la implantación del injerto. Estos factores podrían ser útiles para decidir la retirada del catéter en el mismo acto quirúrgico del trasplante.

En nuestro centro un factor determinante para la retirada del catéter durante el trasplante es la presencia de una infección activa del orificio de salida. En los pacientes portadores de catéter tras el trasplante, les indicamos continuar con los cuidados habituales del orificio de salida.

De los pacientes estudiados, 5 de ellos (4,9\% del total de los que no se retiraron) presentaron infección del orificio de salida postrasplante, precisando tratamiento antibiótico y en aquellos casos que no evolucionaron satisfactoriamente se procedió a su retirada. Si no existe ningún tipo de complicación y la función renal es estable, se procede a la retirada programada del catéter.

Según los resultados, el tiempo medio hasta la retirada del acceso es de 147,3 días ( $\pm 69,6$ días). Este periodo es ligeramente superior al recomendado en las guías y los observados en la bibliografía ${ }^{1,6,8,9}$. Podríamos pensar que este tiempo de espera (tiempo en riesgo) hasta la retirada definitiva del catéter puede aumentar la incidencia de complicaciones, aunque si nos fijamos en los resultados de otros estudios vemos que no es superior $^{4,5,10,11,14}$.

Como principal limitación en el estudio debemos tener en cuenta que se trata de un estudio retrospectivo. Aunque en nuestra serie encontramos que con la retirada de los catéteres de diálisis peritoneal en el acto del trasplante, la lista de espera quirúrgica para 
colocación/retirada de catéteres de nuestro centro se liberaría anualmente de unas 20-25 intervenciones, son necesarios estudios más amplios, para poder analizar cómo repercute este aspecto sobre la optimización de la lista de espera.

Sería interesante realizar estudios para tratar de identificar variables predictivas de cuáles son los pacientes con más riesgo de necesitar diálisis en el trasplante renal inmediato, como pueden ser tiempo de isquemia del injerto, tiempo en diálisis y/o compatibilidad, etc.

A raíz de los resultados obtenidos, valoramos como beneficioso, tanto para el paciente como para la institución, la retirada del catéter peritoneal en el mismo acto quirúrgico del trasplante renal.

De esta manera, evitaríamos una intervención posterior, con los riesgos que conlleva (no valorados en este trabajo).

\section{Agradecimientos:}

A todos los que conforman la unidad de accesos peritoneales, de trasplante renal y el servicio de cirugía urológica, que han realizado este ingente trabajo clínico, que hemos recogido.

A Valeria por apoyarnos en tiempos más difíciles para la investigación no COVID y a la plataforma de bioestadística y epidemiología del instituto de investigación sanitaria del Principado de Asturias (ISPA).

Recepción: 13-12-20

Aceptación: 15-09-21

Publicación: 30-12-21

\section{Bibliografía}

1. Pérez-Fontán M, Remón-Rodríguez C, Coronel F. Trasplante renal en diálisis peritoneal. Guías de Práctica Clínica en Diálisis Peritoneal. Sociedad Española de Nefrología. 2005;304-20.

2. Andreetta $B$, Verrina $E$, Sorino $P$, Edefonti $A$, Perfumo A, Bassi $S$, et al. Complications linked to chronic peritoneal dialysis in children after kidney transplantation: experience of the italian registry of pediatric chronic peritoneal dialysis. Peritoneal Dialysis International. 1996;16(1):570-3.

3. Palmer JA, Kaiser BA, Polinsky MS, Dunn SP, Braas C, Waltz R, et al. Peritoneal dialysis catheter infections in children after renal transplantation: choosing the time of removal. Pediatr Nephrol. 1994;8:715-8.

4. Warren J, Jones E, Sener A, Drage M, Taqui A, Griffin $S$, et al. Should peritoneal dialysis catheters be removed at the time of kidney transplantation. Can Urol Assoc J. 2012;6(5):376-8.

5. Bakir N, Surachno S, Sluiter WJ, Struijk DG. Peritonitis in peritoneal dialysis patients after renal transplantation. Nephrol Dial Transplant. 1998;13:317883.

6. Díaz-Buxo JA, Walker PJ, Burgess WP, Farmer CD, Chandler JT, Faircloth W, et al. The influence of peritoneal dialysis on the outcome of transplantation. The International Journal of Artificial Organs. 1986;9(5):359-62.

7. Brady HR, Abraham G, Oreopoulos DG, Cardella CJ. Bowel erosion due to a dormant peritoneal catheter in immunosuppressed renal transplant recipients. Peritoneal Dialysis International. 1988;8:163-5.

8. $0^{\prime}$ Donogue $D$, Manos J, Pearson $R$, Scott $P$, Bakran $A$, Jonhson $R$, et al. Continuous ambulatory peritoneal dialysis and renal transplantation: a ten-year experience in a single center. Peritoneal Dialysis International. 1992;12:242-9.

9. Evangelista JB, Bennet-Jones D, Cameron JS, Ogg C, Williams DG, Taube DH, et al. Renal transplantation in patients treated with haemodialysis and short term and long term continuous ambulatory peritoneal dialysis. British Medical Journal. 1985;291:1004-7.

10. Patel S, Rosenthal JT, Hakala TR. Management of the peritoneal dialysis catheter after transplantation. Transplantation. 1983;36:589-90.

11. Winchester JF, Rotellar C, Goggins M, Robino D, Alijani MR,Rakowski TA, et al. Transplantation in peritoneal dialysis and hemodialysis. Kidney International. 1993;43(40):101-5. 
12. García-García G, Harden $P$, Chapman J. El papel global del trasplante renal. Nefrología. 2012; 32(1):1-6.

13. Pérez-Fontán $M$, Rodríguez-Carmona $A$, García $T$, Moncalin J, Oliver J, Valdés F. Renal transplantation in patients undergoing chronic peritoneal dialysis. Peritoneal Dialysis International. 1996;16:48-51.

14. Gokal R, Kost S. Peritoneal dialysis immediately post transplantation. Adv Perit Dial. 1999;15:112-5.

Este artículo se distribuye bajo una Licencia Creative Commons Atribución-NoComercial 4.0 Internacional. https://creativecommons.org/licenses/by-nc/4.0/

\section{Open Access (c) (i) \&}

